\section{Doctor, why do I have varicose veins?}

\section{Alvise Cavallini \\ Private Practitioner, Verona, Italy}

Etiopathogenesis of chronic venous disease (CVD) is almost completely obscure.

The pathophysiology is dominated by lower limbs venous hypertension.

In most cases, venous hypertension is caused by reflux through incompetent valves, disregarding the incompetence origin from a primary valvular failure or secondary to parietal dilation.

I will present you some of the most interesting recent articles on this topic, starting from the review published by Bergan and colleagues in 2006. ${ }^{1}$ These authors emphasized the fact that despite the diversity of signs and symptoms associated with chronic venous disease, it seems likely that they are all related to venous hypertension. Venous pressures in the varicose legs reach higher-than-normal levels and remain elevated for prolonged periods and this is an ambulatory hypertension. Another very important point highlighted in this paper is that laminar shear stress can promote the release of factors that reduce inflammation and the formation of reactive free radicals. By contrast, low or zero shear stress, oscillatory or even turbulent flow, promote an inflammatory and thrombotic phenotype.

Ten years later, other authors ${ }^{2}$ have shown how the surgical suppression of the oscillatory component of reflux modulates the inflammatory phenotype.

Therefore, we can explain to our patient that CVD is the inability of the veins to perform their work, with the appearance of signs and symptoms of the disease; in more technical words: the veins are not able to maintain an adequate transmural pressure (i), therefore creating an ambulatory hypertension (ii) that triggers an inflammatory reaction (iii), which determines the morphological alterations typical of varicose veins (alteration of wall/valves).

Are there any environmental/genetic factors that can predispose to this hemodynamic alteration? Many studies exist, in particular we analyze the Edinburgh vein study $^{3}$ and the Framinghan. ${ }^{4}$ There are nonmodifiable factors such as age, race, sex, greater height. The Edinburgh Vein Study found a significant increase in the prevalence from $11.5 \%$ in individuals aged 18 to 24 years to $55.7 \%$ in the age range 55 to 64 years. According to this study the incidence of CVI was similar in men and women, while data from Framingham study demonstrated a prevalence of $1 \%$ in men versus
$10 \%$ in women $<30$ years of age compared with $57 \%$ and $77 \%$ in men and women over the age of 70 years, respectively. It is not true that there are more women than men suffering from varicose veins, however it is true that women generally express more the symptoms and are more concerned with the appearance, therefore they require more frequently the phlebologist's care. Also, the pregnancy has recently been re-evaluated as a cause. The number of pregnancies did not influence GSV reflux patterns in women with primary varicose veins. ${ }^{5}$ Prolonged standing or sitting posture, estrogen therapy, prior leg injury, obesity are modifiable factors. In particular, obesity was associated with the development of CVI: the 13-year incidence $(95 \% \mathrm{CI})$ was $6.1 \%(3.7 \%-9.6 \%)$ in those who were of normal weight and $23.6 \%(14.2 \%-37.0 \%)$ in obese participants. ${ }^{3}$ However, the most important risk factor remains a family history of varicose veins (VV). Evidence for familiar hereditary factors indicate a genetic basis for risk of varicose vein formation. Children of parents suffering from such disease had a $90 \%$ risk of developing VVs when both parents had the condition. There was a significant risk of developing VVs especially in females, but also in males, when only one parent had VVs. It appears that the trait is autosomal dominant with variable penetrance. ${ }^{6}$ Although we do not know which genes are responsible for the varicose disease yet (those that cause the alteration of the wall and/or valves disfunction), some genes responsible for the expressiveness of the disease have been identified, the genes that predispose the patient to the risk of developing the advanced stages of chronic venous insufficiency; we can therefore have prognostic indications on our patients based on their genotype. Dr. Sibilla will speak about this.

Therefore, we can explain to our patient that: i) genetic basis for risk of varicose vein formation exists; we do not know which genes are responsible for the VVs yet, but we know which genes are responsible for the expressiveness of the disease; ii) a correct lifestyle helps (no estrogen therapy, no obesity, no prolonged standing or sitting posture - use of elastic stocking).

It is well recognized that, in varicose veins, there is reflux with incompetent valves and vein wall dilation and both lead to CVD. But how do the valves become incompetent? Are there primary changes in the structure of the valves making them leaky with progressive reflux, which then induces changes in the venous wall and results in CVD? Are there structural abnormalities in the vein wall near the valve junctions that become dilated, and then valve
Correspondence: Alvise Cavallini, Private Practitioner, Verona, Italy.

E-mail: alvise.cavallini@libero.it

Key words: Patients-phlebologists relationship; questions; Italian Association of Phlebology seminar.

This work is licensed under a Creative Commons Attribution 4.0 License (by-nc 4.0).

(C) Copyright A. Cavallini, 2019

Licensee PAGEPress, Italy

Veins and Lymphatics 2019; 8:7937

doi:10.4081/vl.2019.7937

reflux occurs as a secondary event (epiphenomenon)? Until very recently, asking this question was like asking whether the egg or the chicken came first, now we have some evidence. Because the venous pressure in the lower limbs is increased due to hydrostatic reasons in the upright posture, it has been traditionally believed that reflux develops in a retrograde fashion. In primary venous disease, where the valves are intact, it could be assumed that incompetence or absence of the iliac and common femoral valves are the initiating factors for a retrograde development of reflux. The retrograde development of reflux requires incompetence or absence of valves above the saphenofemoral junction (SFJ), which in turn causes dilatation and valvular incompetence sequentially in the greater saphenous vein (GSV) and its tributaries.

...the saphenous trunks are responsible for a progressive retrograde spreading of varicose veins...

This theory has been found to be inaccurate in a number of patients in whom saphenous reflux exists without SFJ or saphenopopliteal junction (SPJ) incompetence.

Although widely accepted, the saphenocentric theory has been criticized.

In 1870, Callender pointed out that many varicose limbs show a normal great saphenous vein (GSV) trunk. This was confirmed by McPheeters, who demonstrated in 1930 by dynamic venography that saphenous veins (SVs) were competent in $71 \%$ of limbs with early and beginning varicose veins and in $30 \%$ of advanced or moderately advanced cases. One year later, the role of incompetence of the SFJ was questioned by Turner Warwick, who affirmed that it is competent in many varicose limbs. Cotton demonstrated in 1961 that the typical changes of varicose disease, including vein dilation, elongation, and tortuosity, appear first and are greater in saphenous tributaries. He also noted that ...the change in the 
caliber of the GSV is often abrupt and coincides with the point of entry of a large varicose tributary. Finally, in 1967 Fegan affirmed that ... an ascending uniform gradual dilatation of the superficial veins occurs. With the introduction of the echocolor-Doppler technology the evidence of an upward development of VVs has been increasingly numerous. Labropoulos and colleagues in their studies ${ }^{7-9}$ demonstrated that reflux can be isolated in a single venous segment or it can be multifocal, often at different sites that do not communicate with or affect each other. Such data indicate a local or multifocal progression of reflux and would indirectly support that local vein wall changes (weakening of the vein wall theory) are responsible for the development of primary CVD. These studies also raised two fundamental questions: i) what leads to tributary reflux without the presence of axial and retrograde insufficiency? ii) if tributaries or superficial venous segments with reflux are left untreated, do they lead to new or extension of reflux in their communicating superficial (i.e. greater and lesser saphenous) or perforator veins? In 2006 the same Authors, in a study of the venous reflux progression analyzed 90 patients (116 limbs) with CVD that had delayed surgery. The reflux pattern was unchanged in $73.3 \%$ of limbs, but in $11.2 \%$ of limbs there was clinical progression and $26.7 \%$ had CFDUS progression. The progression was most common in the great saphenous vein and tributaries followed by perforators. Extension of pre-existing reflux was found in $14.7 \%$ of limbs (in antegrade and retrograde fashion) and new reflux in $12.1 \%$ of limbs. The Authors concluded that: i) anatomic extension of reflux is frequent and progression is commonly seen after six months from the initial Duplex ultrasound; ii) patients undergoing interventions for varicose veins may require another DU scan if more than 6 months have passed since the original exam (modification of the previously planned intervention may be required). These results were subsequently confirmed by others. Caggiati et al. ${ }^{10}$ analyzed two distinct groups of patients: 82 patients aged 30 years and 183 patients aged 60 years. In the 30-year-old group, limb reflux was present in tributaries $(25 \%)$, non-saphenous superficial veins $(36 \%)$ and in saphenous vein (39\%). In the 60-year-old group, reflux in the saphenous vein was more common (62\%) and reflux in the tributaries was frequently associated with reflux in the saphenous vein. It was concluded that the presence of saphenous vein reflux is not required for primary varicose vein pathology to occur.

Also, the different patterns observed in younger $v s$ older patients raise the hypothesis that CVD may progress in an anterograde fashion, starting in the tributaries and affecting the saphenous vein. The same authors had previously shown that the wall of saphenous accessory veins is thinner and contains less muscle than the GSV and that the saphenous fascia would preserve the saphenous vein from excessive pathological dilatation, as a sort of mechanical shield. These anatomic findings could also explain why greater dilatation and tortuosity occur in the saphenous tributaries in primary varicosity. Stretching of the wall of the saphenous compartment as a result of muscular contraction would modify the saphenous vein caliber and consequently modulate the blood flow within it, as happens in the deep veins. ${ }^{11,12}$ An ultrasound study ${ }^{13}$ performed on women with varicose veins (CEAP C2 class) but without edema, skin changes, or ulcers (C3 to C6). Reflux predominated in calf venous segments rather than in the thigh or SFJ. The prevalence of GSV single or multiple segmental reflux without junction involvement (53\%) was significantly higher than the remaining types of reflux. Treatment in most of these patients could be limited to the varicose veins. Correction of SFJ reflux may be needed in $<12 \%$ of the extremities, and only about one third CEAP C2 limbs may require treatment of a refluxing GSV in the thigh.

Hemodynamic principles suggest that primary venous insufficiency follows the hydrostatic column of venous pressure of the limbs, and therefore, venous reflux begins at the lower points and rises upwards. To test the hypothesis of an ascending development of reflux, in 2010 Bernardini et al. ${ }^{14}$ carried out an observational study to analyze the natural evolution of lower limb venous insufficiency. Over a 9-year period, patients with primary superficial venous disease who refused treatment were followed prospectively. In all the worsened refluxes, an extension to reach one or more venous segments at an upper level, uninvolved before, was found. There was no downward-oriented pattern of progression. The authors concluded that natural history of primary venous insufficiency is that of a progressive disease, which begins at lower levels of the limbs and develops in an anterograde manner as venous stasis is higher where force of gravity is higher. These data do not support the aggressive and widespread treatment of terminal valve as first approach. 3 years later, Chastanet and Pittaluga reported similar results. ${ }^{15}$ Varicose veins without saphenous reflux occurred at a younger age (43 versus 55.6 years $\mathrm{P}<0.05)$. In their study the presence of incompetence at the saphenofemoral junc- tion occurred in older patients (58.5 versus 54.1 years $\mathrm{P}<0.05)$. Venous reflux to the ankle also occurred in older patients (mean 64.0 years $\mathrm{P}<0.05$ ). Saphenous vein reflux without clinical varices was associated with more advanced signs (C4-C6: 20.3\% $\mathrm{P}<0.05)$. A younger age was associated with less advanced signs (C0-C2:49.4 versus $\mathrm{C} 4$ C6: 60.1 years $\mathrm{P}<0.05)$. The presence of symptoms was associated with advancing patient age (51.1 versus 49.1 years $\mathrm{P}<0.05$ ). The authors hypothesize that these findings support the concept of early treatment of venous insufficiency before symptomatic and physiological deterioration occurs.

Therefore, primary venous reflux in both superficial and deep veins of the lower limb can take place in an ascending fashion (not retrograde), and importantly the process can be local and segmental. The local development of the disease would suggest that there are susceptible sites, where wall changes, hemodynamic changes, or both occur to initiate reflux. Therefore, the question is, what local factors in the vein wall would cause the secondary segmental reflux and valve dysfunction? Many studies highlight several important points in varicose vein formation: imbalance of connective tissue matrix (collagen; elastin); imbalance of proteolytic tissue degradation; venous segments that are normal but in continuity to varicose veins have the same biochemical properties of the vein wall as varicose veins, suggesting that vein wall changes precede valvular changes; the apoptotic index is $48 \%$ in control veins and only $15 \%$ in varicose veins. Abnormal apoptosis in venous disease disrupts this balance, and favors accumulation of cellular elements and matrix in the wall structure of varicose veins. Inflammation plays a fundamental role, there is much evidence in this regard, and metalloproteinases (MMPs) are the most incriminated mediators. MMPs may have multiple roles that are temporally dependent on the stage of varicose vein development. In the early stages, MMPs may cause venous dilation by inducing hyperpolarization of the vein wall, and inhibiting proper and balanced production of collagen subtypes.

In later stages of CVD, MMPs may alter the vein wall matrix composition to such an extent that dilation and tortuosity become the prevailing morphological feature that is observed clinically in CVD. In conclusion, we could answer to our patient: i) the pathogenesis of varicose vein formation is a complex process and likely multifactorial; ii) it appears that the changes associated with incompetent valves are secondary to alterations in the vein wall; iii) reflux appears to be a local or multifocal process in addition 
to or separate from a retrograde process.

But we did not really answer the question; patients are not interested in the mechanisms of formation of VVs; they want to know why they have developed VVs; what does it really mean?

Varicose veins are common. According to the Edinburgh Vein Study, ${ }^{3}$ approximately one-third of subjects between 18 and 64 years of age have large trunk varicosities, and more than $80 \%$ have smaller reticular or intradermal varices; based on these definitions and demographic data, one can argue about the fact that varicose veins are (at least statistically) normal. If more than half of the population has varicose veins, is it accurate to characterize them as abnormal or even, as some assert, a disease? Why do so many people have these abnormal veins? If varicose veins are abnormal, are they necessarily bad? Is it possible that varicose veins are more benign than generally assumed or perhaps paradoxically beneficial in some circumstances? Conventional wisdom maintains that varicose veins are a disease. Is this true? Are varicose veins always a bad thing? Or does the tendency to produce varicosities reflect traits that might be useful, or perhaps even valuable, under certain circumstances?

Collateral vessels (especially superficial ones) that form around a region of venous obstruction (typically deep), are usually described as secondary varicose veins. These are healthy physiological adaptations to unexpected venous obstruction and are therefore considered to be good things (even when they produce some of the same undesirable signs associated with primary varicose veins.); no one wants primary varicose veins, but many people (i.e. those with chronic venous obstruction) welcome secondary (collateral) varicose veins if they prevent a stasis ankle skin ulcer from occurring. People who develop primary varicose veins should also be good at developing collateral (secondary) varicose veins when necessary. Curiously, this observation the best varicose vein makers are likewise the best collateral varicose vein makers is not well described in the literature. Yet, in retrospect, it seems almost obvious that this must be the case (or, to skeptics, perhaps it seems too good to be true?).

And is there a relationship between varicose veins and arterial collaterals? It has been reported that varicose veins are less common in patients undergoing infrainguinal arterial bypass; one explanation for this observation is that patients with pri- mary varicose veins are better at making arterial collaterals and are therefore less likely to need bypass surgery than those with varicose veins.

The Normative Aging Study ${ }^{16}$ demonstrated a $36 \%$ decreased risk of symptomatic coronary disease in patients with varicose veins (compared with those without) along with a highly significant increase in survival-free of coronary heart disease in patients with varicose veins. There are reports confirming a clinical association between varicose veins and certain forms of lymphatic proliferation; for example, lymphedema distichiasis (a condition in which lymphatic vessels proliferate excessively to the detriment of the patient) has been shown to be associated with an increased tendency for varicose veins (and that both conditions may be affected by the FOXC2 gene). (Primary) varicose veins may not be a disease, and they are not caused by degeneration of normal veins. They are caused by genetic factors that work to actively drive the development of new veins and/or venous remodeling that results in vein enlargement, wall changes, etc. The same genetic factors that lead to varicose vein formation may also promote the formation of venous, arterial, and lymphatic collateral vessels. Just as people differ in their (genetic) tendencies to make varicose veins, they likewise differ in their ability to make other types of collateral vessels. People with varicose veins may have lower morbidity/mortality from vascular diseases than do those without varicosities; perhaps is it because they are better at making venous, arterial, and lymphatic collateral vessels? People hate varicose veins. They are unattractive and, in many cases, symptomatic. But there may be an upside to their presence if they reflect factors that predispose to lower cardiovascular/lymphatic morbidity and mortality. Varicose veins may therefore be a marker of one's vasculogenic capacity and paradoxically good things to have. Might there be a positive side to varicose veins? Now there's a message of hope that many patients will be glad to hear.

\section{References}

1. Bergan JJ, Schmid-Schönbein GW, Smith PD, et al. Chronic venous disease. N Engl J Med 2006;355:488-98.

2. Zamboni P, Spath P, Tisato V, et al. Oscillatory flow suppression improves inflammation in chronic venous disease. J Surg Res 2016;205:238-45.

3. Lee AJ, Robertson LA, Boghossian SM, et al. Progression of varicose veins and chronic venous insufficiency in the general population in the Edinburgh Vein Study. J Vasc Surg Venous Lymphat Disord 2015;3:18-26.

4. Brand FN, Dannenberg AL, Abbott RD, Kannel WB. The epidemiology of varicose veins: the Framingham Study. Am J Prev Med 1988;4:96-101.

5. Engelhorn CA, Cassou MF, Engelhorn AL, Salles-Cunha SX. Does the number of pregnancies affect patterns of great saphenous vein reflux in women with varicose veins? Phlebology 2010;25: 190-5.

6. Raffetto JD, Mannello F. Pathophysiology of chronic venous disease. Int Angiol 2014;33:212-21.

7. Labropoulos N, Giannoukas AD, Delis $\mathrm{K}$, et al. Where does venous reflux start? J Vasc Surg 1997;26:736-42.

8. Labropoulos N, Kang SS, Mansour MA, et al. Primary superficial vein reflux with competent saphenous trunk. Eur J Vasc Endovasc Surg 1999;18:2016.

9. Labropoulos N, Leon L, Kwon S, et al. Study of the venous reflux progression. J Vasc Surg 2005;41:291-5.

10. Caggiati A, Rosi C, Heyn R, et al. Agerelated variations of varicose veins anatomy. J Vasc Surg 2006;44:1291-5.

11. Mazayshvili K. The superficial venous pump. Vein and Lymphatics 2018;7: 7280 .

12. Caggiati A. Fascial relationships of the long saphenous vein. Circulation 1999; 100:2547-9.

13. Engelhorn CA, Engelhorn AL, Cassou MF, Salles-Cunha SX. Patterns of saphenous reflux in women with primary varicose veins. J Vasc Surg 2005; 41:645-51.

14. Bernardini E, De Rango P, Piccioli R, et al. Development of primary superficial venous insufficiency: the ascending theory. Observational and hemodynamic data from a 9-year experience. Ann Vasc Surg 2010;24:709-20.

15. Chastanet S, Pittaluga P. Patterns of reflux in the great saphenous vein system. Phlebology 2013;28:39-46.

16. Scott TE, Mendez MN, LaMorte WW, et al. Are varicose veins a marker for susceptibility to coronary disease? Results from the normative aging study. Ann Vasc Surg 2004;18:459-64. 